\title{
Hemangiopericytoma in the Retroperitoneal Space: A Case Report
}

\author{
Authors \\ Dr Dreamy Jain ${ }^{1}$, Dr S.H. Khaparde ${ }^{2}$ \\ ${ }^{1}$ Junior Resident, ${ }^{2}$ Professor and Head, \\ Department Of Pathology, P.D.V.V.P.F's Medical College, Ahmednagar
}

\begin{abstract}
Introduction: A hemangiopericytoma is a rare soft tissue tumor of vascular origin representing $1 \%$ of all vascular neoplasms. It is derived from a pericyte of Zimmerman, which is a modified smooth muscle cell that surrounds the small blood vessels. Hemangiopericytoma can occur wherever there are vascular capillaries. In this paper, we report a case of hemangiopericytoma in retroperitoneal space.

Case Presentation: A 30-year-old male presented with a palpable lump on the left abdomen. Preoperative imaging indicated that the tumor was in the retroperitoneal space without invasion of other organs. A complete resection was performed via a midline incision. The histological and immunohistochemical staining patterns supported the diagnosis of a hemangiopericytoma.

Conclusion: Complete resection is the best way to treat a hemangiopericytoma. Despite its rare incidence in comparision with other mesenchymal tumors of retroperitoneal space, hemangiopericytoma should be considered in the differential diagnosis of well defined lobulated retroperitoneal masses.
\end{abstract}

\section{Introduction}

A hemangiopericytoma is a rare soft tissue tumor of vascular origin representing $1 \%$ of all vascular neoplasms. It is derived from a pericyte of Zimmerman, which is a modified smooth muscle cell that surrounds the small blood vessels. This type of tumor was first described by Stout and Murray in $1942^{[1]}$. It tends to be large and well encapsulated $^{[2]}$. It represents approximately $5 \%$ of all sarcomatous tumors, and can occur anywhere, but more usually in the extremities, retroperitoneum, pelvis (uterus, ovary, and urinary bladder), head, neck and lungs. ${ }^{[3]}$. Approximately $25 \%$ of hemangiopericytomas arise from the retroperitoneum and pelvic cavity ${ }^{[4]}$. We describe a rare case of a hemangiopericytoma in the retroperitoneal space.

\section{Case Presentation}

A 30-year-old male presented with a palpable lump on the left side of abdomen for the past six months. He had no pain or tenderness associated with the lesion, and no other clinical symptoms. On physical examination, the lump was firm with a smooth surface. Rest of the clinical examination was unremarkable. Blood examination was within normal limits. Computed tomography (CT) scan of abdomen showed a well defined mass in the 
retroperitoneal space. Magnetic resonance imaging (MRI) showed that the outer layer was heterogenous with high intensity and that the central layer had an extremely high intensity in T2-weighted images, indicating that the inside of the mass had a rich blood flow.

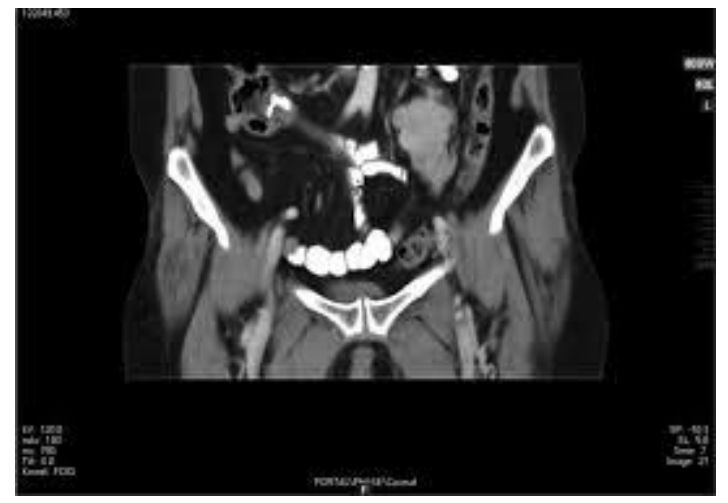

Figure1. Computed tomography scan of hemangiopericytoma

The preoperative images indicated that the tumor was derived from the retroperitoneum. Because of its vascularity, the pre-operative diagnosis was a soft-tissue tumor such as a solitary fibrous tumor, fibrous histiocytoma, synovial sarcoma or hemangiosarcoma. A biopsy was avoided due to the risk of needle track seeding. The patient underwent complete excision of tumor mass via midline incision. The excised tumor was $8.5 \times 6 \times 4.5 \mathrm{cms}$ in diameter with a capsule. Its cut surface was grey-white and at places hemorrhagic areas were also seen.

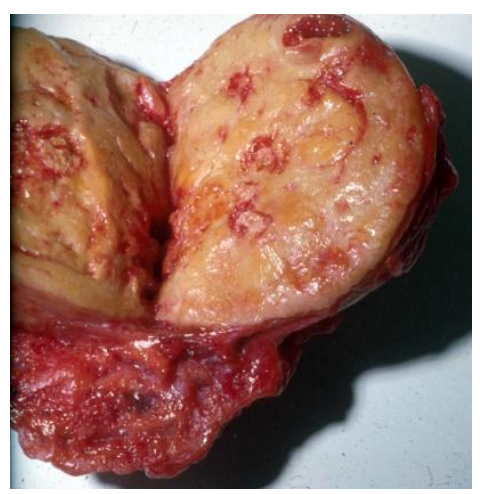

Figure 2:_Gross image of hemangiopericytoma

Microscopically, the tumor showed vascular channels lined with endothelium. These vascular spaces are surrounded by tumor cells with round to oval nuclei and a moderate amount of eosinophilic cytoplasm.

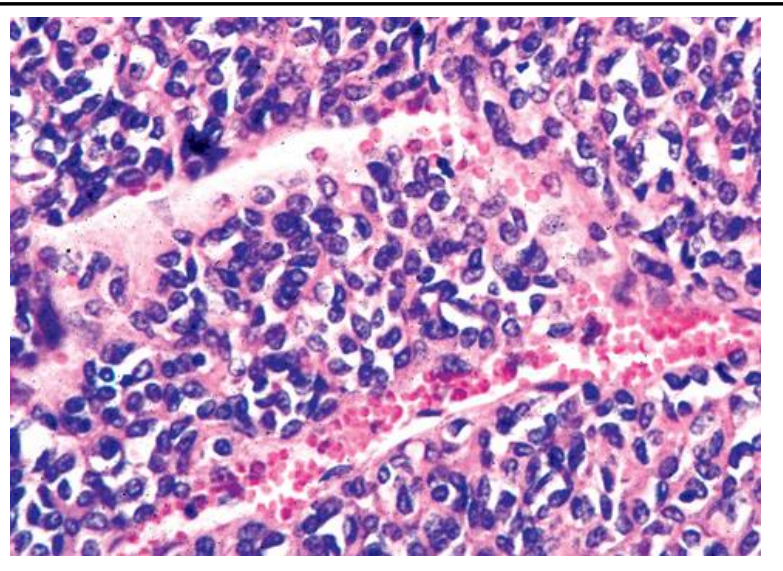

Figure 3: Hematoxylin and eosin staining revealing tumor cells surrounding endotheliallined vascular spaces.

Spindle cells were also noted at places. There were focal hemorrhages and a few mitotic figures(1-2/10HPF). The histopathological picture of the tumor was compatible with hemangiopericytoma. Furthermore, immunohistochemical stains for actin, desmin, S-100, Bcl2 and CD99 were negative while stains for viamentin and CD34 were positive. The tumor was pathologically diagnosed as hemangiopericytoma.

\section{Discussion}

Little has been published about hemangiopericytoma, a rare soft tissue tumor. It can occur anywhere vascular capillaries are found. The tumors most commonly occurs in the musculature of the extremities, retroperitoneum, pelvis ( uterus, ovary, and urinary bladder), head, neck and lungs ${ }^{[3]}$.It has an equal sex distribution and occurs between $4 \& 72$ years of age ${ }^{[4]}$.

The pathological diagnosis of a hemangiopericytoma, in comparision to other mesenchymal tumors such as solitary fibrous tumors, can be difficult ${ }^{[4]}$. Bcl-2 and CD99 Iimmunohistochemistry were used to distinguish a hemangiopericytoma from a solitary fibrous tumor because a solitary fibrous tumor is positive for Bcl-2 and CD99 ${ }^{[5],[6]}$. We diagnosed a hemangiopericytoma following an examination of the structural features of the mass. Spindle shaped cells surrounding the endothelial-lined vascular 
spaces were observed by H\&E staining, and the mass was positive for vimentin and negative for Bcl-2 \&CD99.

Radiotherapy and chemotherapy are not generally effective for the treatment of a hemangiopericytoma ${ }^{[7]}$. Some have advocated the use of adjuvant radiotherapy in response to locally aggressive nature of hemangiopericytoma but, due to tumor radioresistance, no differences in local disease control were observed between treatment with and without adjuvant radiotherapy ${ }^{[8]}$. Spitz et al. reported that hemangiopericytomas showed a poor response to chemotherapy. They treated six patients with pre-operative chemotherapy, and only one of them responded to anthracyclinebased chemotherapy ${ }^{[9],[10]}$. Therefore, complete surgical resection is the only effective therapy for hemangiopericytoma.

Spitz et al. also reported that 5-year and 10-year survival rates of patients with a hemangiopericytoma were $71 \%$ and $54 \%$, respectively. Since many benign and malignant diseases occur in the retroperitoneal space, recognizing the presence of this malignant tumor in the retroperitoneal space is important for management.

\section{Conclusion}

This report presented a rare case of a hemangiopericytoma in the retroperitoneal space. Despite its rare incidence in comparision with other mesenchymal tumors of retroperitoneal space, hemangiopericytoma should be considered in the differential diagnosis of well defined lobulated retroperitoneal masses.

\section{References}

1. Hart S,Sisley L. Hemangiopericytoma of the presacral space.Br J Surg 1973, 60: 583-584. PubMed Abstract I Publisher Full Text

2. Goldman SM, Davidson AJ. Retroperitoneal \& pelvic hemangiopericytoma, clinical, radiologic and pathologic correlation. Radiology 1988; 168: 13-17.
3. Vennarecci G, Boschetto A, Giovannelli L, Buscaglia F, Corazza V, Santoro R, Mancini P, Lorusso R, Marino M, Ettorre GM: Malignant hemangiopericytoma of the mesorectum.

4. Chir Ital 2004, 56: 865-868. Pub Med Abstract

5. Enzinger FM, Smith BH, Hemangiopericytoma : an analysis of 106 cases, Hum Pathol 1976;7; 61-82. PubMed Abstract I Publisher Full Text

6. Fukunaga M, Naganuma H, Ushigome S, Endo Y, Ishikawa E: Malignant solitary fibrous tumor of the peritoneum.

7. Histopathol 1996, 28: 463-466. Publisher Full Text

8. Cristi E, Perrone G, Battista C, Benedetti Panici P, Rabitti C: A rare case of solitary fibrous tumor of the presacral space: morphological and immunohistochemical features.

9. In Vivo 2005, 19:777-780 PubMed Abstract I Publisher Full Text

10. del Rosario ML, Saleh A: Preoperative chemotherapy for congenital hemangiopericytoma and a review of the literature. 